\title{
LETTERS
}

\section{Centralized referral systems can both shorten wait times and preserve patient autonomy}

In their commentary, Urbach and Martin highlight the issue of access to elective surgeries during the coronavirus disease 2019 (COVID-19) crisis. $^{1}$ However, their solution - a single-entry care model - is overly simplistic and will do little to address patient wait times for many elective surgeries, such as cataract surgery. Even before COVID-19, surgeons across Canada had unacceptably long wait times - more than 1 year in several regions.

A single-entry model would be useful in hospitals where surgeons performing the same procedure have large variations in wait times. But even in this scenario, patients having elective procedures like cataract surgery may want to preserve some choice. A more reasonable solution would be a centralized referral system so that patients can choose either the shortest wait time or a specific surgeon. I perform cataract surgery out of St. Mary's General Hospital in Kitchener, Ontario, where all 9 cataract surgeons have unacceptably long wait times. To address this, before COVID-19, we worked with regional partners to adopt a centralized referral system that would allow referring providers to see surgeon wait times. We also published periodic hospital newsletters highlighting current surgeon wait times for cataract surgery. These solutions empower the patient and referring provider to use wait-time information to influence their choice about the surgeon without removing their autonomy.

An essential COVID-19-related issue for elective surgery not addressed in the commentary is the compounding problem of how we are to maintain operating room efficiencies. Before COVID-19, my hospital provided excellent and efficient care with 18 cataract surgeries completed per day, but this required patients to congregate in open-concept preoperative and postoperative areas. How can we maintain these efficiencies with the new need for social distancing and repeatedly sanitized surfaces? If we can't, we may be facing a 2-year average wait for a procedure that patients require to maintain visual function and driving standards.

\section{Chryssa N. McAlister MD MHSc}

Ophthalmologist and co-division lead for ophthalmology, St. Mary's General Hospital, Kitchener, Ont.

Cite as: CMAJ 2020 June 15;192:E659. doi: $10.1503 / \mathrm{cmaj} .75698$

\section{Reference}

1. Urbach DR, Martin D. Confronting the COVID-19 surgery crisis: time for transformational change. CMAJ 2020;192:E585-6.

Competing interests: None declared. 